\title{
A conserved role of Parkinson-associated DJ-1 metabolites in sperm motility, mitosis, and embryonic development
}

Susanne Bour ${ }^{1, *}$, Yanina Dening $2,3,{ }^{*}$, Melanie Balbach${ }^{4}$, Ina Poser5, Inés Ramírez Álvarez ${ }^{2,10}$, Helena den Haan ${ }^{6}$, Christoph Kluge ${ }^{7}$, Ronald Naumann ${ }^{5}$, Reinhard Oertel $^{8}$, Irene Alba-Alejandre ${ }^{9}$, Davide Accardi ${ }^{5, \gamma}$, Christian G. Stief ${ }^{1}$, Marianne Dieterich $^{2,10}$, Peter Falkai ${ }^{3}$, Rainer A. Böckmann7, Horacio Pérez-Sánchez ${ }^{6}$, Anthony A. Hyman ${ }^{5}$, Matthias Trottmann ${ }^{1}$ and Francisco Pan-Montojo ${ }^{2,3,10, \Psi}$

1 Department of Urology, University Hospital, LMU Munich, Marchioninistr. 15, 81377 Munich

2 Department of Neurology, University Hospital, LMU Munich, Marchioninistr. 15, 81377 Munich

3 Department of Psychiatry, University Hospital, LMU Munich, Nußbaumstr. 7, 80336 Munich

${ }^{4}$ Department of Pharmacology, Weill Cornell Medical College, New York City, NY

5 Max-Planck Institute for Cell Biology and Genetics, Pfotenhauerstr. 108, 01307 Dresden

${ }^{6}$ Bioinformatics and High Performance Computing Research Group (BIO-HPC), Computer Engineering Department, Universidad Católica de Murcia (UCAM), 30107 Murcia, Spain.

7 Computational Biology, Department of Biology, Friedrich-Alexander University, Staudtstr. 5, 91058 Erlangen

${ }^{8}$ Institute of Clinical Pharmacology, Medical Faculty, Technical University Dresden Fiedlerstrasse 27, 01307 Dresden Germany

${ }^{9}$ Department of Gynecology and Obstetrics, Ludwig-Maximilian University Munich, Maistr. 11, 80337 Munich

10 Munich Cluster for Systems Neurology (SyNergy)

${ }^{*}$ These authors contributed equally to this work.

$\gamma$ Current address: Champalimaud Centre for the Unknown, Av. Brasilia, Doca de Pedroucos 1400-038 Lisbon, Portugal

$\Psi$ Corresponding author:

Dr. Francisco Pan-Montojo

Department of Psychiatry

University Hospital, LMU Munich

Nußbaumstr. 7

80336 Munich

francisco.pan-montojo@med.uni-muenchen.de 


\begin{abstract}
Fertility rates in the developing world have dramatically dropped in the last decades. This drop is likely due to a decline in sperm quality and women having children at older ages. Loss of function mutations in $D J-1$, a Parkinson's associated gene, are linked to alterations in multiple cellular processes such as mitochondrial activity, ROS production or sperm motility and lead to an early onset of Parkinson's disease and male infertility in humans and other species. Glycolate (GA) and D-lactate (DL), products of DJ-1 glyoxalase activity, sustain mitochondrial function and protect against environmental aggressions. We, therefore, tested whether these substances could also have a rescue effect on these phenotypes. Here, we show that $D J-1$ loss of function not only affects sperm motility but also leads to defects in mitosis and an age-dependent increase in the abortion rate. Remarkably, whereas DL was only able to rescue embryonic lethality in $C$. elegans, GA rescued these phenotypes in all model systems tested and even increased sperm motility in wild-type sperm. These positive effects seem to be mediated through an increase in NAD(P)H production and the regulation of intracellular calcium. These findings not only strongly suggest GA as a new therapeutic candidate to improve male and female fertility but also show its potential to treat diseases associated with a decline in mitochondrial function or to improve mitochondrial function in aging.
\end{abstract}

\title{
Introduction
}

Fertility rates in the developing world have dramatically dropped in the last decades (reviewed in ${ }^{1}$ ). This drop is likely due to a decline in sperm quality, specially a reduction in spermatozoa concentration and motility, and a shift in the mean age at which women decide to start having children. Alterations in sperm quality and increases in the abortion rate have been related to aging and to exposures to environmental toxins. Therefore, looking for treatments that could improve sperm quality and increase fertility protecting against environmental agressions is of the essence.

Loss of function mutations in DJ-1 (also known as PARK-7) are associated with mitochondrial dysfunction leading to early onset of familial Parkinson's disease (PD) ${ }^{2}$. These mutations have also been associated with defects in sperm motility and male infertility in humans and other species ${ }^{3-5}$. At the cellular level, $D J-1$ loss of function mutations are associated with abnormal mitochondrial morphology and dynamics, increased sensitivity to oxidative stress, opening of the 
mitochondrial permeability transition pore, and alterations in calcium homeostasis ${ }^{6-8}$. Despite intensive research in the last decade to elucidate the molecular mechanisms underlying this plethora of cellular functions, they still remain unknown.

DJ-1 has recently been identified as a member of a novel glyoxalase familiy ${ }^{8}$. Two systems of glyoxalases have been described: 1) Glutathione-dependent Glo I and Glo II systems (GLOD-4) ${ }^{9}$ and 2) cofactor-independent Glo III system (DJ-1) ${ }^{10 .}$ Glyoxalases are enzymes that transform 2-oxoaldehydes (i.e. glyoxal and methylglyoxal) into their corresponding 2-hydroxyacids (i.e. glycolate (GA) and D-lactate (DL)). Glyoxal and methylglyoxal are potent aldehydes, covalently reacting with proteins and lipids to form advanced glycation end-products (AGEs), which have been implicated in PD and many other neurodegenerative diseases 11,12. Thus, DJ-1 and GLOD-4 were considered to act as detoxifying proteins. GA and DL were first described more than a century ago as a component of the sugar cane by Shorey ${ }^{13}$ and a product of glycolysis and amino acid metabolism by Dakin and Dudley ${ }^{14}$, respectively, but their function remained unknown for a long time. The results of our previous study suggested that these glyoxalases have two different but complementary functions: They detoxify harmful substances produced by glycolysis (i.e. glyoxal and methylglyoxal) and they produce GA and DL, two substances that support mitochondrial function. We have shown that knocking down $D J-1$ in HeLa cells and $C$. elegans leads to alterations in the mitochondrial membrane potential and increased sensitivity to oxidative stress caused by environmental toxins such as paraquat ( $\mathrm{PQ}$, a widely used pesticide associated with the appearance of PD) or desiccation ${ }^{15}$. Interestingly, these phenotypes were rescued by the co-treatment with the products of $D J-1, \mathrm{GA}$ and $\mathrm{DL}$, in a dose-dependent manner. Moreover, the addition of these substances to dopaminergic neurons in vitro had a neuroprotective effect against PQ.

Low levels of $D J-1$ or its deletion have been associated with male infertility in humans, flies and mice, mostly due to defects in sperm motility ${ }^{3-5}$. In this paper we show that knocking down djr-1.1/djr-1.2 or glod-4 in worms and $D J-1$ in mice did induced a reduction in the brood size of worms and in the litter size in mice. Interestingly, this reduction was not only due to a decrease in sperm motility but also to an age-dependent increase in the abortion rate, which is normally due to alterations in embryonic development. Treatment with GA or DL in worms and with GA, but only partially with DL, rescued these phenotypes in HeLa cells, sperm and worms and increased sperm motility even in wild type (WT) sperm. 


\section{Materials \& Methods}

\section{Chemicals}

HBSS without $\mathrm{Ca}^{2+}$ and $\mathrm{Mg}^{2+}$, PBS, poly-L-lysine, poly-D-lysine, $\mathrm{CaCl}_{2}$, glycolic acid, D-lactate, HEPES, ionomycin, Glutamic acid, hydrochloric acid solution, glucose (all from Sigma Aldrich, Germany, EU), DNAse I (Roche, Germany, EU). High glucose Dulbecco's Modified Eagle Medium (DMEM, 31966-021), FBS, Pen/Streptomycin, trypsin, Fluor 4-AM, TMRE and Rhod-2 AM (all from ThermoFischer Scientific, USA). Anhydrous DMSO and Pluronic F-127 (Sigma Aldrich, Germany) were diluted at a $20 \%(\mathrm{w} / \mathrm{v})$ and used to reconstitute Fluo-4 AM stocks right before use.

\section{Ethical statement}

All animal procedures were performed according to the German Law for Animal Experiments (Tierschutzversuchsordnung). The ethical committee from the Munich University Hospital approved all procedures on human sperm (Az: 50514).

\section{Determination of mouse litter size and abortion rate}

To determine the abortion rate 8 to 30 weeks-old C57Bl6/J and PARK-7 $\%$ mice ( $n=16$, matching ages) were sacrificed between day E14.5 and E15.5 and the number of embryos and abortions was determined. On a second batch of animals, litter size was determined as the number of newborn mice per pregnant mice $(n=15)$.

\section{Determination of embryonic lethality and brood size}

All C. elegans strains were maintained on NGM agar plates seeded with Escherichia coli NA22 at $15^{\circ} \mathrm{C}$. Wild type (N2) and mutant strains $\Delta \Delta d j r$ and glod$4(\mathrm{tm} 1266)$ were obtained from Prof. Kurzchalia's laboratory at the Max Planck Institute for Cell Biology and Genetics. The procedures to obtain the DJ-1 double mutant mice have been already described ${ }^{15}$. To determine embryonic lethality, individual adult worms from each strain were transferred to a 6-well plate well with NGM and E. coli (NA22) (with or without GA or DL) to lay eggs. After 4 hours, adult worms were removed and the number of laid eggs was counted. The percentage of hatched eggs was calculated (L1/(L1+remaining eggs)*100) 8 hours after removing the adults. To determine brood size, L4 worms were transferred to a 6-well plate well with NGM and NA22 and allowed to lay eggs. To prevent confusion, adult worms were transferred to another well every 24 hours until no laid eggs could be observed. The number of L4 and adult worms in each well were counted 24 hours after the adult worm had been transfered, and the brood size was determined for each strain.

\section{Determination of cell growth}

Cell growth was determined by two different methods. The first method (WST1Assay) was used to analyze cell growth at different time points using the same 
plates: 500 cells of 8 different PARK7 KO clones and HeLa Kyoto wild type cells were seeded in 96 well plates ( 6 wells / line). For each time point ( $0 \mathrm{~h}, 48 \mathrm{~h}$, $122 \mathrm{~h}$, and $144 \mathrm{~h}$ ), WST1 was added to the cells according to the manufacturer's instructions and incubated for $30 \mathrm{~min}$ at $37^{\circ} \mathrm{C}$. Absorbance was measured at $450 \mathrm{~nm}$ and $620 \mathrm{~nm}$ using an EnVision Plate Reader (PerkinElmer).

The second method was used to analyze the rescue effect of GA and DL. Briefly, HeLa cells were seeded and treated with medium containing distilled water, 5 mM GA, or $5 \mathrm{mM}$ DL. 48 hours later, the number of living cells was calculated with the help of an automated cell counter (ThermoFischer, USA).

\section{CRISPR}

HeLa-Kyoto PARK7 KO clones had been kindly provided by Martin Stewart (Koch Institute, MIT, Cambridge, USA). Briefly, cells were electroporated with the NEON device (Invitrogen) using a sgRNA-Cas9-NLS complex targeting human PARK7 at exon 1 (sg1: CTCGCCTCATGACATCTACAGGG; sg2: AGATGTCATGAGGCGAGCTGGGG). Subsequently, cells were seeded in serial dilution and clones were characterized by genotyping, sequencing, and Western blot.

\section{Quantitative Western blot (anti PARK7):}

Western blot was performed using anti-PARK7/DJ1 (sc-32874, SantaCruz) and anti-Tubulin (T9026, Sigma) antibodies and analyzed using an Odyssey imaging system (Licor).

\section{Semen sample collection and motility measurements}

Mouse sperm samples were obtained from the epididymis of sacrificed C57Bl/6J or PARK-7-/- mice ( $\mathrm{n}=3$ per group). Briefly, both epididymis of each male were placed in $200 \mu \mathrm{l}$ of an isotonic $0.9 \% \mathrm{NaCl}$ solution and fat tissue was removed. Epididymis were then placed into $170 \mu \mathrm{l} \mathrm{CPA}$ buffer (18\% raffinose and 3\% milk powder in distilled water), cut into several pieces, and left at RT for 5 min to allow the spermatozoa to swim into the media. $4 \mu \mathrm{l}$ of the sperm- containing suspension was then pipetted into $196 \mu \mathrm{l}$ HTF medium supplemented with 10 $\mathrm{mM}$ of GA or DL or an equivalent amount of $\mathrm{NaCl}$ in distilled water to control for osmolality (vehicle) and incubated at $37^{\circ} \mathrm{C}$ for $60 \mathrm{~min}$ before starting the measurements. Mouse sperm motility measurements were performed using the Hamilton Thorne IVOS platform (Hamilton Thorne, USA) and the specific sperm motility analysis software for mice. Briefly, the IVOS platform was warmed up to $37^{\circ} \mathrm{C}$ before starting the measurements. $25 \mu \mathrm{l}$ of sperm samples in HTF medium from each treatment group were loaded on a Leja-slide and introduced in the machine. After focusing on the samples, the machine analyzed 10 fields per samples. This analysis was performed before and 5, 30, 60 and 90 minutes after addition of vehicle, GA, or DL.

Frozen bull semen samples were provided from Swiss genetics, Switzerland. Human sperm samples were collected from patients enrolled in the study 
attending the diagnostic Department of the Urologic Clinic of the LudwigMaximilians University Munich for Semen Diagnostics and Artificial Insemination. All patients were between 28 and 58 years of age. Semen samples of 68 normozoospermic healthy patients were obtained by masturbation after 3 to 5 days of sexual abstinence. Some of the samples were diluted in SpermFreeze buffer (LifeGlobal, USA) and stored in liquid nitrogen for later analysis.

Fresh human samples were left at $37^{\circ} \mathrm{C}$ for 2 hours before semen samples were gently centrifuged ( $800 \mathrm{x} \mathrm{g}, 4 \mathrm{~min}$ ) and resuspended in sperm iTALP buffer ( 99 $\mathrm{mM} \mathrm{NaCl}, 3.1 \mathrm{mM} \mathrm{KCl}, 25 \mathrm{mM} \mathrm{NaHCO}, 0.35 \mathrm{mM} \mathrm{NaH}_{2} \mathrm{PO}_{4}-\mathrm{H}_{2} \mathrm{O}, 0.5 \mathrm{mM}$ HEPES, $2.0 \mathrm{mM} \mathrm{CaCl} 2-2 \mathrm{H}_{2} \mathrm{O}, 1.1 \mathrm{mM} \mathrm{MgCl} 2-6 \mathrm{H}_{2} \mathrm{O}$ and $\mathrm{H}_{2} \mathrm{O}$ dest, pH 7.4), containing $0.25 \mathrm{mg} / \mathrm{ml}$ Polivinylalcohol (PVA) . On the day of analysis, frozen human and bull sperm samples were thawed at $37^{\circ} \mathrm{C}$, centrifuged $(800 \times \mathrm{g}, 4$ min), and the supernatant was exchanged with iTALP buffer $\mathrm{pH}$ 7.4. After resuspension in iTALP buffer, human (fresh and thawed) and bull semen samples $(\mathrm{n}=10)$ were divided into treatment groups (vehicle (distilled water with $\mathrm{NaCl}$ ), $30 \mathrm{mM}$ or $60 \mathrm{mM}$ GA with or without $1,3,5$ and $10 \mu \mathrm{M}$ of CatSper channel blocker NCC55-0396). Sperm motility was blindly and manually assessed following the WHO guidelines (2010) before treatment and 5, 30, 60, and $90 \mathrm{~min}$ after treatment. Mitochondrial activity was analysed by Fluorescent Activated Cell Sorter as described below.

\section{Fluorescence Activated Cell Sorting (FACS) of semen subpopulations}

Semen samples were adjusted to $2.0-4.0 \times 10^{8} / \mathrm{ml}$ and stained with JC-1 (2 $\mu \mathrm{l} / \mathrm{ml}$ ) in iTALP buffer for $10 \mathrm{~min}$ at RT. The samples were analyzed with a Becton Dickinson BD FACS Calibur Flow Cytometer. For detection we used the following settings: FSC (E00, 9.99 linear), SSC (510 mV, 1.00, linear), FL1 (480 $\mathrm{mV}, 1.00$, logarithmic) with $488 \mathrm{~nm}$ wavelength and FL2 (520 mV, 1.00, logarithmic) with $543 \mathrm{~nm}$ wavelength. For each sample, 20,000 cells were counted. Compensation between two channels was adjusted to $59.9 \%$. Mitochondrial activity was evaluated by fluorescence emission of JC-1, which emits green fluorescence when the membrane potential is depolarized and red fluorescence when the membrane potential shifts to more negative values. Populations of cells were separated in depolarized and polarized mitochondrial membrane potential subpopulations by adjusting compensation and laser power in a way that two populations could be observed. For analysis, Flowing Software 2.0 (University of Turku, Finland) was used. Subpopulations were gated and analyzed accordingly.

\section{NAD(P)H live-cell microscopy on HeLa cells}

$\mathrm{NAD}(\mathrm{P}) \mathrm{H}$ live-cell microscopy of HeLa cells was performed as previously described ${ }^{16}$. Briefly, NAD(P)H fluorescence intensity time series were performed on a ZEISS LSM880 inverted confocal equipped with an incubation chamber to maintain 37 Celsius degree and $5 \%$ of CO2. Fluorophores were excited using a $355 \mathrm{~nm}$ UV laser (Coherent), while the fluorescent signal was detected using a 
GaAsP spectral detector narrowing down the band of absorption between 455 and $473 \mathrm{~nm}$. To maximize the transmission efficiency of the system in excitation and detection and reduce the aberrations due to the watery environment, a ZEISS Plan C-ApoChromat 40x/1.2 Water lens with depth compensating correction collar was used. In addition, bright field images were taken using a HeNe 633 laser as source of light and a T-PMT to detect the signal. The sampling factor in XY (pixel size) of each image was equal to $208 \mathrm{~nm}$, which lead to a final resolution of approximately $600 \mathrm{~nm}$. For each image a volume of $5 \mu \mathrm{m}$ around the specimen central plane was taken by acquiring 3 planes separated by a Z-step of $2.5 \mu \mathrm{m}$. Time series measurements were obtained with 5 min time resolution. Fluorescence intensity levels were extracted using FIJI Image Analysis Freeware

\section{Analyzing changes of the intracellular $\mathrm{Ca}^{2+}$ concentration in sperm and HeLa cells using a fluorescence plate reader}

Mouse sperm were isolated by incision of the cauda epididymis followed by a swim-out in modified TYH medium (135 mM NaCl, $4.8 \mathrm{mM} \mathrm{KCl}, 2 \mathrm{mM} \mathrm{CaCl}{ }^{2}, 1.2$ $\mathrm{mM} \mathrm{KH2PO4,} 1 \mathrm{mM} \mathrm{MgSO}{ }^{4}, 5.6 \mathrm{mM}$ glucose, $0.5 \mathrm{mM}$ sodium pyruvate, $10 \mathrm{mM} \mathrm{L-}$ lactate, $10 \mathrm{mM}$ HEPES, pH 7.4). After $15 \mathrm{~min}$ swim-out at $37^{\circ} \mathrm{C}$, sperm were collected and counted. For capacitation, mouse sperm were subsequently incubated for $90 \mathrm{~min}$ in TYH containing $3 \mathrm{mg} / \mathrm{ml} \mathrm{BSA}$ and $25 \mathrm{mM} \mathrm{NaHCO}$. Human sperm were purified by a "swim-up" procedure in human tubular fluid (HTF): $97.8 \mathrm{mM} \mathrm{NaCl}, 4.69 \mathrm{mM} \mathrm{KCl}, 0.2 \mathrm{mM} \mathrm{MgSO}_{4}, 0.37 \mathrm{mM} \mathrm{KH}_{2} \mathrm{PO}_{4}, 2.04 \mathrm{mM}$ $\mathrm{CaCl}_{2}, 0.33 \mathrm{mM}$ Na-pyruvate, $21.4 \mathrm{mM}$ lactic acid, $2.78 \mathrm{mM}$ glucose, and $21 \mathrm{mM}$ HEPES, pH 7.4. For capacitation, human sperm were incubated for $3 \mathrm{~h}$ in HTF containing $3 \mathrm{mg} / \mathrm{ml}$ human serum albumin and $25 \mathrm{mM} \mathrm{NaHCO}$. Changes in $\left[\mathrm{Ca}^{2+}\right]_{\mathrm{i}}$ were measured in 384-microtiter plates in a fluorescence plate reader (Fluostar Omega, BMG Labtech, Germany) at $30{ }^{\circ} \mathrm{C}$. Mouse and human sperm were loaded with Cal-520-AM ( $5 \mu \mathrm{M}$, Molecular Probes, USA) in the presence of Pluronic F-127 $(0.02 \% \mathrm{v} / \mathrm{v})$ at $37^{\circ} \mathrm{C}$ for $45 \mathrm{~min}$. After incubation, excess dye was removed by centrifugation ( $700 \mathrm{xg}, 7 \mathrm{~min}, \mathrm{RT})$. Each well was filled with $50 \mu \mathrm{l}$ of the sperm suspension $\left(5 \cdot 10^{6} \mathrm{sperm} / \mathrm{ml}\right)$; fluorescence was excited at $480 \mathrm{~nm}$, emission was detected at $520 \mathrm{~nm}$ with bottom optics. After 10 cycles, the experiment was interrupted and $10 \mu \mathrm{l}$ stimuli (mouse sperm: $10 \mathrm{mM} \mathrm{GA} / 9 \mathrm{mM}$ $\mathrm{NaCl} / 10 \mathrm{mM}$ 8-Br-cAMP, human sperm: $30 \mathrm{mM}$ GA/27 mM NaCl/2 $\mu \mathrm{M}$ of progesterone) or buffer as control were added to the sperm suspension using an electronic multichannel pipette. After 60 cycles, $10 \mu \mathrm{l} 2 \mathrm{mM} \mathrm{CaCl}^{2}$ or buffer as control were added to each well.

Fluorescence measurements on HeLa cells were carried out in 96 well-plates (FBottom, Greiner, Germany, EU) using the fluorescence plate-reader Fluostar Omega (BMG Labtech, Ortenberg) with a 485/12 nm excitation filter with bottom optics for excitation and the Em520 nm filter for emission. $30.000 \mathrm{HeLa}$ cells were plated in each well and left 12 hours at $37^{\circ} \mathrm{C}$ and $5 \% \mathrm{CO}_{2}$ in the incubator overnight. On the next day, cells were loaded with Fluo-4 AM or TMRE as described for calcium imaging during mitosis. $20 \mathrm{~min}$ after the last HBBS change, cells were exposed to either $4,5 \mathrm{mM} \mathrm{NaCl}$ as osmotic control or GA $(2,5$ 
and $5 \mathrm{mM}$ ) after $5 \mathrm{~min}$ by adding $10 \mu \mathrm{l}$ extra HBBS containing the substances (10x concentrated, $10 \%$ of the total volume) and $2 \mu \mathrm{M}$ Ionomycin $20 \mathrm{~min}$ later.

\section{Results:}

\section{Knocking down $D J-1$ leads to a reduction of progressive sperm motility, an increased abortion rate and alterations in cell proliferation}

First we investigated the effect of knocking down $D J-1$ in mice and djr1.1/djr1.2 or glod-4 in worms on litter and brood sizes. We used DJ-1 KO (PARK-7\%-) mice, djr-1.1;djr-1.2 double $C$. elegans mutants lacking both $D J-1$ orthologues ( $\Delta \Delta d j r)$, and glod-4 C. elegans mutants lacking the Glo I/II system. Our results show that the loss of $D J-1$ in mice leads to a significant decrease in the litter size (Figure 1a). In worms, the loss of djr-1.1 and djr-1.2 and to a lesser extent the loss of glod-4 leads to a significant reduction of the brood size (Figure 1b). To investigate whether this phenotype was due to alterations in sperm motility and/or embryonic development, we analyzed sperm motility in mice and the abortion rate/embryonic lethality in mice and worms respectively. As expected, we observed a reduction in the percentage of rapidly progressive motile sperm (Grade A motility according to the world health organization (WHO)) between sperm isolated from DJ-1 KO mice and WT mice (Figure 1c). Interestingly and unexpected, we also observed an age-dependent increase in the abortion rate in $D J-1$ KO female mice compared to WT females (Figure $1 \mathrm{~d}$ and 1e). This finding was also reproduced in $C$. elegans, where an increase in embryonic lethality in $\Delta \Delta-d j r$ and glod-4 worms (measured as a reduction in the percentage of hatched eggs) compared to the control (Figure 1f) was observed.

It has been reported that, in HeLa cells, knocking down $D J-1$ alters the rounding force exerted when the cells round-up to prepare and perform mitosis. The generation of a rounding force during mitosis is important for the transitions between different mitotic phases. This is also crucial during embryonic development ${ }^{17}$. To elucidate whether knocking down $D J-1$ also lead to a defect in mitosis and, thereby, cell proliferation, we analyzed cell proliferation in WT and DJ-1 KO HeLa cells, generated by CRISPR/Cas9. Our results show a significant delay in the growth curve of DJ-1 KO HeLa cells when compared to WT (Figure $1 \mathrm{~g})$. Interestingly, this delay varied between the eight different $D J-1$ KO clones (Figure 1h), suggesting that subtle gene-environment interactions had an important effect on this phenotype. 


\section{Glycolic acid and (partially) D-lactate increase sperm motility and rescue both the embryonic lethality and the cell proliferation phenotypes:}

We then tested whether treatment with GA or DL, the products of DJ-1 and GLO could rescue the phenotypes observed in DJ-1 KO mice and $\Delta \Delta$-djr and glod-4 worms. We first analyzed the effect of $10 \mathrm{mM} \mathrm{GA}$ or DL on WT and DJ-1 KO mouse sperm motility. Adding GA, but not DL, to cauda sperm rescued the defect in Grade A rapidly progressive sperm motility in $D J-1 \mathrm{KO}$ mice (Figure 2a) and had a positive effect on overall sperm motility (increase in the percentage of spermatozoa with Grade A+B motility) also on WT mice (Figure 2b). The positive general effect of GA on mouse sperm motility was also shown for bull and human sperm using 30 and $60 \mathrm{mM}$ GA (Figure 2c), demonstrating that the effect of GA on sperm Grade A+B motility is conserved between species. Remarkably, this effect was maintained over time for at least $90 \mathrm{~min}$ in all species (Figures 2d-h).

The effect of GA and DL on embryonic development was tested on $\Delta \Delta$-djr and glod-4 worms and their effect on cell proliferation in HeLa cells. Both GA and DL treatments rescued the embryonic lethality phenotype in worms (Figure 3a) whereas only the addition of GA, but not DL, rescued the cell proliferation phenotype of HeLa cells (Figure $3 \mathrm{~b}$ ). Altogether, these results show that loss of DJ-1 affects progressive, sperm motility, embryonic development, and cell proliferation, and that these cellular defects can be rescued by the addition of GA and, in some phenotypes, DL.

\section{Glycolic acid and D-lactate decrease intracellular calcium and glycolic acid, but not D-lactate, increases mitochondrial energy production}

Based on our previous results showing that GA and DL supported mitochondrial membrane potential, we first analyzed whether the effect on sperm motility was mediated by an increase in mitochondrial activity. The mid piece of the sperm flagellum contains mitochondria, which together with glycolysis has a role in energy production ${ }^{18}$ ensuring reproductive success. Sperm progressive motility has been correlated with mitochondrial membrane potential $\left(\psi_{\mathrm{m}}\right)$ and consequently with mitochondrial functionality: an increase in $\psi_{\mathrm{m}}$ is directly proportional to an increase in progressive motility and oxygen consumption in human sperm ${ }^{19}$. In clinical studies, mitochondrial function is generally monitored using cationic fluorescent probes such as MitoTracker or JC-120. Using JC-1, Paoli et al. were able to demonstrate that both human sperm motility and viability are associated with $\psi_{\mathrm{m}}{ }^{21}$. The authors found that the higher the fluorescence signal, the better the membrane integrity of the mitochondria and a higher motility. Therefore, we stained human spermatozoa with JC-1 to analyze the effect of GA on the $\psi_{\mathrm{m}}$. The percentage of sperm with active mitochondria 
between GA-treated and control samples did not differ at the beginning. If something, treatment with $60 \mathrm{mM}$ GA caused a very small but significant decrease in the percentage of bull and human sperm with higher fluorescence values (Figure 4a-c). Thus, an improvement of the mitochondrial membrane integrity does not seem to underlie the increase in progressive motility after GA treatment. We therefore looked for other factors that could improve sperm motility.

Sperm motility also relies on a tight regulation of intracellular $\mathrm{Ca}^{2+}$ levels ${ }^{22}$. The principal $\mathrm{Ca}^{2+}$ channel in mammalian sperm is the CatSper channel, specially in humans. Human CatSper is activated upon depolarization of the membrane potential and alkalization of the intracellular $\mathrm{pH}$, but also by extracellular ligands, acting as a polymodal, chemosensory $\mathrm{Ca}^{2+}$ channel that plays a vital role in the regulation of sperm hyperactivation ${ }^{23-25}$. In the absence of CatSper, sperm do not hyperactivate and cannot fertilize the oocyte, resulting in male infertility ${ }^{25}$. In addition, it has been suggested that the mobilization of intracellular $\mathrm{Ca}^{2+}$ stores through inositol 3-phosphate receptors (InsP3Rs) 26,27 and Ryanodine receptors (especially RyR 1 and 2) ${ }^{28}$ located at the acrosome and the sperm neck, respectively, also control sperm function. To test whether the effect of GA on sperm motility is dependent on $\mathrm{Ca}^{2+}$ influx through CatSper, we first used a pharmacological approach. Human and bull sperm were treated with NNC55-0396, which inhibits CatSper channel function already at low $\mu \mathrm{M}$ concentrations ${ }^{24,29}$. In human sperm, treatment with $1.5,3,5$ or $10 \mu \mathrm{M}$ NNC550396 reduced sperm motility and co-treatment with GA rescued this effect in all cases except for the $10 \mu \mathrm{M}$ concentration (Figure $4 \mathrm{~d}$ ). In contrast and surprisingly, NNC55-0396 had no negative effect on bull sperm motility and GA increased sperm motility in its presence (Figure 4e). The positive effect of GA on human sperm motility in the presence of the blocker suggests that the effect of GA was independent of CatSper. We then tested the effect of GA on intracellular calcium. Sperm cells were loaded with Cal520-AM and the changes in fluorescence were recorded using a plate reader before and after the addition of GA. Addition of GA to mouse sperm did not evoke an increase in $\left[\mathrm{Ca}^{2+}\right]_{i}$ as observed in WT mice exposed to 8-Br-cAMP, which directly activates CatSper (Figure 4f). If something, GA induced a non-specific but significant reduction in $\left[\mathrm{Ca}^{2+}\right]_{\mathrm{i}}$ when compared to control sperm. This same effect could also be observed in the absence of CatSper in sperm from CatSper KO mice (Figure 4h). Again suggesting that this effect was independent of CatSper. Similar to mouse sperm, in human sperm, addition of GA evoked a non-specific but significant reduction in $\left[\mathrm{Ca}^{2+}\right]_{\mathrm{i}}$ (Figure $4 \mathrm{~g}$ ). Thus, whereas it is unclear how the small but significant reduction in $\left[\mathrm{Ca}^{2+}\right]_{i}$ would lead to an increase in sperm motility (discussed in detail below), the increase in sperm motility caused by GA in human and mouse sperm cannot be explained by an increase in $\left[\mathrm{Ca}^{2+}\right] \mathrm{i}$. 
The next logical step would have been to measure energy production in sperm cells. However, this is a complicated assay and it is unclear how informative the results from such an assay would have been. This is so because, even if GA increases energy production in sperm, it also increases sperm motility, which is associated with a higher energy consumption. Therefore, we tested the effect of GA and DL on energy production in HeLa cells. Our previous results had shown that $D J-1$ loss of function altered mitochondrial membrane potential (increased in worms and decreased in HeLa cells) and GA and DL were able to rescue this phenotype. However, as in the case of sperm cells, these results gave no real information as to whether these substances have a positive effect on energy production. In HeLa cells NAD(P)H levels can be measured using NAD(P)H auto fluorescence ${ }^{16}$ (Figure 5a). As HeLa cells do not move, one would not expect any alterations in energy consumption in these cells by adding GA or DL as in the case of spermatozoa. Our results show that in these cells, GA but not DL, increased NAD(P)H levels (see Figure 5b).

Finally, based on the results obtained on $\left[\mathrm{Ca}^{2+}\right]_{\mathrm{i}}$ in sperm, we tested the effect of GA and DL on the $\left[\mathrm{Ca}^{2+}\right]_{\mathrm{i}}$ in HeLa cells. We charged HeLa cells with the $\mathrm{Ca}^{2+}$ fluorescent marker Fluo-4 AM and analyzed the changes in $\left[\mathrm{Ca}^{2+}\right]_{\mathrm{i}}$ caused by the addition of GA , DL or vehicle. In these cells, the addition of $5 \mathrm{mM}$ (or higher) but not $2,5 \mathrm{mM}$ of GA or DL resulted in a significant decrease of $\left[\mathrm{Ca}^{2+}\right]_{i}$ when compared to the osmotic control ( $\mathrm{NaCl}$ ) (Figure $5 \mathrm{c}$ and $\mathrm{d}$ ).

\section{Discussion:}

Alterations in sperm motility and/or embryonic development lead to a reduction in fertility. In this study we show that knocking down DJ-1 reduced the litter size in mice and knocking down djr-1.1/djr-1.2 or glod-4 reduced the brood size in worms. In mice, the litter size reduction was associated with a reduction in sperm motility and surprisingly to an age-dependent increase in the abortion rate, which had not been previously described. These findings were reproduced in C. elegans. In this model organism, the brood size reduction was also associated to an increase in embryonic lethality.

Abortion and embryonic lethality are associated to alterations in embryonic development. During embryonic development, cells constantly divide and migrate. The migration process is associated with different kinds of forces exerted on the cells. This forces also affect embryonic cell division ${ }^{30}$. A recent article showed that DJ-1 is involved in mitotic rounding and the absence of DJ-1 leads to a reduction in the force exerted during mitotic rounding in the initial phases of mitosis ${ }^{17}$. To divide, most animal cells drastically change shape and round up against extracellular confinement. This process is even more important during embryonic development, as embryonic cells need to be able to counteract 
external migrating forces to divide properly. Therefore, we analysed whether knocking down $D J-1$ in HeLa cells would lead to alterations in cell proliferation. Indeed, our results showed that knocking-down $D J-1$ in HeLa cells leads to a reduction in cell proliferation.

In general and under controlled environmental conditions, the mutation penetrance of knocking down DJ-1 in mice and HeLa cells or its orthologs or glod-4 in worms suggest that gene-enviroment interactions play an important role in the development of these phenotypes. Knocking-down $D J-1$ in mice or in HeLa cells induced an overall mild negative effect (15-20\% difference to wildtype for most of the values measured) and, in the case of abortion, appeared only in older animals. In HeLa cells, the overall effect on cell proliferation was mild even with some clones showing no clear phenotype. djr-1.1/djr-1.2 and glod-4 KO worms only exhibited a mild impairment in embryonic development. In worms the effect of these mutations on sperm motility remains to be further analyzed.

These results correlate well with previous published studies that analysed the phenotype of DJ-1 KO mice showing no neurodegeneration or any other clear phenotype apart from a mild alteration in the mitochondrial function and an increase in glutathione peroxidase activity ${ }^{31}$. They also correlate well with our previous results showing that knocking out djr1.1/djr1.2, glod-4, or all three genes together in C. elegans did not have any obvious effect on the life cycle of the worm under normal environmental conditions, apart from increasing the mitochondrial membrane potential ${ }^{15}$. Interestingly, under stress conditions, the penetrance dramatically increased. The lack of these genes in worms caused: i) a decreased tolerance to desiccation and posterior rehydration with mortality rates up to $80 \%$ when compared to $\mathrm{N} 2$ worms and associated with the disruption of the mitochondrial network ${ }^{15}$ and ii) a decreased tolerance to PQ exposure, also increasing mortality rates. Thus, further underlining the importance of gene-environment interactions. These phenotypes were stronger in the triple mutant compared to $\Delta \Delta$ djr1.1/djr1.2 or glod-4 double or single mutants, suggesting that the remaining gene/-s were able to partially compensate the loss of the other gene/-s. Desiccation/rehydration of worms can be observed when the soil dries out and loses humidity. Under these conditions, worms protected themselves by up-regulating the expression of djr-1.1, djr1-2, and glod-4 (among other genes) in a process called preconditioning that takes place when the worms sense a reduction in room humidity ${ }^{32}$. This up-regulation seems to be important to prepare for rehydratation, where a boost in the production of glyoxal and methylglyxoal can be expected and the up-regulation of these genes should lead to an increase in GA and DL levels. In that study, we also showed that feeding GA and DL to the mutant worms was able to rescue these phenotypes. Thus, suggesting that an increase of GA and DL levels is part of 
the survival strategy of $C$. elegans in dry environments and is required to overcome and protect against the stress induced by this metabolic stop/start, a process that could be compared to ischemia/reperfusion damage. A similar protective effect was observed upon exposure to mitochondrial toxins like PQ, that increased mortality rates in mutated worms compared to $\mathrm{N} 2$, and was rescued by the addition of GA and, to a lesser extent, DL. These results were also reproduced in murine dopaminergic neurons in vitro.

In contrast to $C$. elegans, no up-regulation of $D J-1$ or $G L O D-4$ levels has been observed in mammals. In the literature, only a mild up-regulation of GLOD-4 expression in a mouse model of Alzheimer's disease has been described ${ }^{33}$. Despite the inability to regulate this genes, our results show that high concentrations of GA and DL still have neuroprotective properties in mammals. In a recently published study, we showed that GA protects against ischemia in several stroke models: in an oxygen glucose deprivation (OGD) model in vitro, in a global cerebral ischemia mouse model and in a middle cerebral artery occlusion mouse model ${ }^{34}$. We have also been able to reproduce the in vitro results in dopaminergic neurons in a mouse model of PD generated by exposing WT and DJ-1 KO mice to PQ. In this ongoing study, we also observed that knocking down DJ-1 in mice had no effect on the concentration of GA in serum (reference to be added). Considering that GA and DL only exert their effect when using high concentrations of GA and DL (at least orders of magnitude higher than the physiological concentrations detected in the plasma of mice, i.e. $>1 \mathrm{mM}$ vs. $<0.1 \mathrm{mM}$ ), these results suggest that mammals might have lost this gene regulatory pathway during evolution and that the observed rescue and protective effects of high concentration of GA and DL is a conserved evolutionary trait in higher organisms, even if they lost the ability to up regulate the expression of these genes. Supporting this hypothesis, overexpression of $D J-1$ has been described as neuroprotective in several studies using mammalian cell lines ${ }^{35,36}$.

Based on these results, we investigated whether GA and DL could rescue the phenotypes observed in sperm motility, embryonic development or cell proliferation and tried to identify the possible underlying mechanisms. Overall, our results show that GA and DL worked to a different extend depending on the phenotype. Whereas treatment with GA rescued all phenotypes and increased sperm motility even in WT sperm, treatment with DL only had a positive effect on rescuing embryonic development in worms. Our results also suggest that GA exerts its effect through i) a decrease in $\left[\mathrm{Ca}^{2+}\right]_{i}$ and ii) an increase in NAD(P)H production, most probably mitochondrial. This does not seem to be the case for DL, that had a similar effect on $\left[\mathrm{Ca}^{2+}\right]_{\mathrm{i}}$ but showed no effect on NAD(P)H production, suggesting that this unique feature of GA might underlie the differences in their effect on these phenotypes. The reason for this difference 
needs to be further investigated. Interestingly, many neurological diseases are associated with an impaired mitochondrial function and an increased intracellular calcium, thought to play an important role in the pathophysiological process. Therefore, the effects of GA and DL on intracellular calcium and/or mitochondrial function could also explain their neuroprotective properties.

The results also suggest that these two different mechanisms do not need to take place at the same time to elicit a positive effect. It seems that this would depend on the cell type and whether only one or both mechanisms are needed to exert a beneficial effect in that specific cell. For example, in the case of sperm the possible role of reducing $\left[\mathrm{Ca}^{2+}\right]_{\mathrm{i}}$ to enhance sperm motility is unclear. Reducing the concentration of $\mathrm{Ca}^{2+}$ in i-TALP buffer did not increase sperm motility (data not shown). Moreover, calcium influx through CatSper channels and the resulting increase in intracellular $\mathrm{Ca}^{2+}$ are essential for sperm motility ${ }^{24,37}$. Accordingly, blocking CatSper channels with NNC55-0396 reduced human sperm motility. GA was able to counteract the inhibitory effect of NNC55-0396 on sperm motility up to a concentrations of $5 \mu \mathrm{M}$. Thus suggesting that the effect of GA is independent of the regulation of $\left[\mathrm{Ca}^{2+}\right]_{\mathrm{i}}$. This was also the case in bovine sperm, even if, surprisingly, NNC55-0396 did not show any effect on bovine sperm motility. A literature search did not find any studies investigating the effect of NNC55-0396 on bovine sperm motility, so it could be that NNC55-0396 does not affect bovine sperm motility. Another argument in this direction is the lack of other sources of $\mathrm{Ca}^{2+}$ in sperm cells. Mammalian sperm cells only contain a reminiscent of the ER and the presence of other $\mathrm{Ca}^{2+}$ channels (e.g. ER-related), apart from CatSper channels, is still a matter of debate $26,27,37-39$. Therefore, it seems more plausible that the positive effect of GA on sperm motility is due to an increase in energy production. In this regard, JC-1 staining did not have any clear effect on mitochondrial membrane potential. The next logical step would have been to compare ATP or NAD(P)H production between control and GA treated sperm. These experiments are complicated to perform and we believe that, in the specific case of GA, that also enhances sperm motility, it would have been difficult to extract a conclusion from such measurements. This is so because GA also increases sperm motility, which would also increase energy consumption. This has been shown in capacitated sperm. Capacitation dramatically increases sperm motility and energy consumption which is then partially compensated by a boost of energy production from glycolysis and mitochondrial oxidative phosphorylation (OXPHOS), the two major metabolic pathways producing ATP in sperm (reviewed in ${ }^{40}$ ). The fact that only GA but not DL can increase sperm motility also suggests that increases in mitochondrial energy production would be more important than $\mathrm{Ca}^{2+}$ regulation in exerting this effect. This assumption is based on the data obtained with HeLa cells. As mentioned above, our results show that GA reduces $\left[\mathrm{Ca}^{2+}\right]_{\mathrm{i}}$ and increases NAD(P)H production in this cell type. The distribution pattern of the NAD(P)H signal obtained with the confocal 
microscope, similar to the pattern of the mitochondrial network, suggests that $\mathrm{NAD}(\mathrm{P}) \mathrm{H}$ had a mitochondrial origin. On the other hand, DL only decreased intracellular $\mathrm{Ca}^{2+}$ having no effect on energy production.

A review of the literature shows how $\left[\mathrm{Ca}^{2+}\right]_{\mathrm{i}}$ and energy availability also play an important role in embryonic development, mitosis and how alterations in these processes can have severe consequences and result in several diseases ${ }^{41-43}$. It is therefore not surprising that, by modulating $\left[\mathrm{Ca}^{2+}\right]_{i}$ and enhancing energy production, GA and DL have such an effect not only in these cellular functions but also rescuing pathological conditions such as neurological diseases. Further studies are necessary to understand how reducing $\left[\mathrm{Ca}^{2+}\right]_{\mathrm{i}}$ and increasing energy production rescue the effect of knocking down $D J-1$ on mitosis and embryonic development and whether this would also be beneficial in other diseases or in aging.

Overall the results of this study could have far-reaching implications, not only in the field of neurology, reproduction and embryonic development, but also in other diseases or in aging, where alterations in calcium homeostasis and mitochondrial dysfunction or insufficient energy production are common underlying pathophysiological features.

Competing interest: FP-M has one patent on the effect of glycolic acid on sperm motility and filed a patent on the effect of glycolic acid on energy production. The rest of the authors declare no competing interest.

Author's contribution list: FP-M conceived and designed the study. FP-M planned and perform the experiments in mice. SB, YD, RN, IA, MT and FP-M designed and performed the sperm motility experiments. MB designed and performed the calcium measurements in sperm. IP, IR-A, YD, AAH and FP-M planned and performed the HeLa experiments. FP-M planned and performed $\mathrm{C}$. elegans embryonic lethality experiments. IR-A, YD, SB, MT, IP, HvH, CK, RB, RO, HP-S, MD, CGS, MB, AAH and FP-M analyzed the data. MB, IP, SB, IR-A, MT, HP-S and FP-M wrote the manuscript and all other authors critically corrected and reviewed the manuscript.

\section{Acknowledgements:}

This work was funded by the Deutsche Forschungsgemeinschaft (DFG, German Research Foundation) under Germany's Excellence Strategy within the framework of the Munich Cluster for Systems Neurology (EXC 2145 SyNergy - ID 390857198). We would like to thank Dr. Viktoria Ruf for her help with the fluorescence plate reader. C. elegans mutant lines were generated and kindly provided by Prof. Teymuras Kurzchalia and Dr. Cihan Erkut. Prof. Teymuras Kurzchalia was also of great help critically revising the manuscript. CRISP/Cas-9 
modified HeLa Park-7\%- clones were kindly provided by Dr. Martin Steward from the MIT. Dr. Ünal Coskun was of great help critically reviewing the manuscript and proposing additional critical experiments. Thanks to Wolfgang John and Jussi Helppi from the MPI Animal Facility for their help with PARK-7 mice. We would also like to thank the animal caretakers at the Zentrum für Neuropathologie und Prionforschung, especially Dr. G. Mitteregger, Fang Zhang, Heike Jäckle and Tanja Simon for their help with the animals.

\section{References:}

1. Skakkebaek, N.E., et al. Male Reproductive Disorders and Fertility Trends: Influences of Environment and Genetic Susceptibility. Physiological reviews 96, 55-97 (2016).

2. Bonifati, $V_{\text {., }}$ et al. Mutations in the DJ-1 gene associated with autosomal recessive early-onset parkinsonism. Science 299, 256-259 (2003).

3. An, C.N., et al. Down-regulation of DJ-1 protein in the ejaculated spermatozoa from Chinese asthenozoospermia patients. Fertil Steril 96, 19-23 e12 (2011).

4. Hao, L.Y., Giasson, B.I. \& Bonini, N.M. DJ-1 is critical for mitochondrial function and rescues PINK1 loss of function. Proc Natl Acad Sci U S A 107, 9747-9752 (2010).

5. Yoshida, K., et al. Immunocytochemical localization of DJ-1 in human male reproductive tissue. Mol Reprod Dev 66, 391-397 (2003).

6. Irrcher, I., et al. Loss of the Parkinson's disease-linked gene DJ-1 perturbs mitochondrial dynamics. Hum Mol Genet 19, 3734-3746 (2010).

7. Giaime, E., Yamaguchi, H., Gautier, C.A., Kitada, T. \& Shen, J. Loss of DJ-1 does not affect mitochondrial respiration but increases ROS production and mitochondrial permeability transition pore opening. PloS one 7, e40501 (2012).

8. Shtifman, A., Zhong, N., Lopez, J.R., Shen, J. \& Xu, J. Altered Ca2+ homeostasis in the skeletal muscle of DJ-1 null mice. Neurobiol Aging 32, 125-132 (2011).

9. Thornalley, P.J. Glyoxalase I--structure, function and a critical role in the enzymatic defence against glycation. Biochem Soc Trans 31, 1343-1348 (2003).

10. Misra, K., Banerjee, A.B., Ray, S. \& Ray, M. Glyoxalase III from Escherichia coli: a single novel enzyme for the conversion of methylglyoxal into Dlactate without reduced glutathione. Biochemical Journal 305, 999-1003 (1995).

11. Castellani, R., Smith, M.A., Richey, P.L. \& Perry, G. Glycoxidation and oxidative stress in Parkinson disease and diffuse Lewy body disease. Brain Res 737, 195-200 (1996).

12. Li, J., Liu, D., Sun, L., Lu, Y. \& Zhang, Z. Advanced glycation end products and neurodegenerative diseases: mechanisms and perspective. J Neurol Sci 317, 1-5 (2012).

13. Shorey, E.C. GLYCOLLIC ACID: ONE OF THE ACIDS OF SUGAR-CANE. Journal of the American Chemical Society 21, 45-50 (1899). 
14. Dakin, H.D. \& Dudley, H.W. THE INTERCONVERSION OF $\alpha$-AMINO-ACIDS, $\alpha$-HYDROXY-ACIDS AND $\alpha$-KETONIC ALDEHYDES. PART II. Journal of Biological Chemistry 15, 127-143 (1913).

15. Toyoda, Y., et al. Products of the Parkinson's disease-related glyoxalase DJ-1, D-lactate and glycolate, support mitochondrial membrane potential and neuronal survival. Biol Open (2014).

16. Blacker, T.S., et al. Separating NADH and NADPH fluorescence in live cells and tissues using FLIM. Nat Commun 5, 3936 (2014).

17. Toyoda, Y., et al. Genome-scale single-cell mechanical phenotyping reveals disease-related genes involved in mitotic rounding. Nat Commun 8, 1266 (2017).

18. Visconti, P.E. Sperm bioenergetics in a nutshell. Biol Reprod 87, 72 (2012).

19. Moscatelli, N., et al. Single-cell-based evaluation of sperm progressive motility via fluorescent assessment of mitochondria membrane potential. Sci Rep 7, 17931 (2017).

20. Amaral, A. \& Ramalho-Santos, J. Assessment of mitochondrial potential: implications for the correct monitoring of human sperm function. Int J Androl 33, e180-186 (2010).

21. Paoli, D., et al. Mitochondrial membrane potential profile and its correlation with increasing sperm motility. Fertil Steril 95, 2315-2319 (2011).

22. Singh, A.P. \& Rajender, S. CatSper channel, sperm function and male fertility. Reprod Biomed Online 30, 28-38 (2015).

23. Lishko, P.V., Botchkina, I.L. \& Kirichok, Y. Progesterone activates the principal Ca2+ channel of human sperm. Nature 471, 387-391 (2011).

24. Strunker, T., et al. The CatSper channel mediates progesterone-induced Ca2+ influx in human sperm. Nature 471, 382-386 (2011).

25. Ren, D., et al. A sperm ion channel required for sperm motility and male fertility. Nature 413, 603-609 (2001).

26. Walensky, L.D. \& Snyder, S.H. Inositol 1,4,5-trisphosphate receptors selectively localized to the acrosomes of mammalian sperm. J Cell Biol 130, 857-869 (1995).

27. Ho, H.C. \& Suarez, S.S. Hyperactivation of mammalian spermatozoa: function and regulation. Reproduction 122, 519-526 (2001).

28. Harper, C.V. \& Publicover, S.J. Reassessing the role of progesterone in fertilization--compartmentalized calcium signalling in human spermatozoa? Hum Reprod 20, 2675-2680 (2005).

29. Mannowetz, N., Miller, M.R. \& Lishko, P.V. Regulation of the sperm calcium channel CatSper by endogenous steroids and plant triterpenoids. Proc Natl Acad Sci U S A 114, 5743-5748 (2017).

30. Heisenberg, C.-P. \& Bellaïche, Y. Forces in Tissue Morphogenesis and Patterning. Cell 153, 948-962 (2013).

31. Andres-Mateos, E., et al. DJ-1 gene deletion reveals that DJ-1 is an atypical peroxiredoxin-like peroxidase. Proceedings of the National Academy of Sciences 104, 14807-14812 (2007).

32. Erkut, C., et al. Molecular strategies of the Caenorhabditis elegans dauer larva to survive extreme desiccation. PloS one 8, e82473 (2013). 
33. Hoos, M.D., et al. Longitudinal study of differential protein expression in an Alzheimer's mouse model lacking inducible nitric oxide synthase. $J$ Proteome Res 12, 4462-4477 (2013).

34. Chovsepian, A., et al. Glycolic acid protects neurons against ischemia $<$ em>in vitro</em> and in two animal models of stroke. bioRxiv, 2020.2011.2024.396051 (2020).

35. Oh, S.E. \& Mouradian, M.M. Cytoprotective mechanisms of DJ-1 against oxidative stress through modulating ERK1/2 and ASK1 signal transduction. Redox Biol 14, 211-217 (2018).

36. Johnson, W.M., et al. Regulation of DJ-1 by Glutaredoxin 1 in Vivo: Implications for Parkinson's Disease. Biochemistry 55, 4519-4532 (2016).

37. Alasmari, W., et al. Ca2+ signals generated by CatSper and Ca2+ stores regulate different behaviors in human sperm. J Biol Chem 288, 6248-6258 (2013).

38. Florman, H.M., Jungnickel, M.K. \& Sutton, K.A. Regulating the acrosome reaction. Int J Dev Biol 52, 503-510 (2008).

39. Costello, S., et al. Ca2+-stores in sperm: their identities and functions. Reproduction 138, 425-437 (2009).

40. Ferramosca, A. \& Zara, V. Bioenergetics of mammalian sperm capacitation. BioMed research international 2014, 902953 (2014).

41. Gyllenhammer, L.E., Entringer, S., Buss, C. \& Wadhwa, P.D. Developmental programming of mitochondrial biology: a conceptual framework and review. Proceedings. Biological sciences 287, 20192713 (2020).

42. Stackley, K.D., Beeson, C.C., Rahn, J.J. \& Chan, S.S. Bioenergetic profiling of zebrafish embryonic development. PloS one 6, e25652 (2011).

43. Paudel, S., Sindelar, R. \& Saha, M. Calcium Signaling in Vertebrate Development and Its Role in Disease. International journal of molecular sciences 19(2018). 


\section{Figure legends:}

Figure 1. Effects of DJ-1 loss of function in embryonic development and cell proliferation. Dot plot graphic in (a) shows the number of E14.5 embryos from WT and DJ-1 KO mice. Dot plot graphic in (b) shows the brood size in $d j-1$ (djr1.1/djr1.2, $\Delta \Delta)$ and glod-4 KO worms. Dot plot graphic in (c) shows the percentage of sperm with progressive rapid motility in WT and DJ-1 KO mice. Dot plot graphic in (d) shows the abortion rate (number of aborted embryos/total number of embryos) in WT and DJ-1 KO mice. Graphic in (e) shows the evolution of the abortion rate with age. Dot plot graphic in (f) shows the abortion rate for WT and DJ-1 KO mice. Dot plot graphic in (1) displays embryonic lethality in WT, $\Delta \Delta$ and glod-4 worms shown as the percentage of hatched eggs to the total of laid eggs. (g) Growth curve of WT and DJ-1 KO HeLa cells during $144 \mathrm{~h}$. Graphic in (h) shows the effect of knocking out DJ-1 in the growth of HeLa clones using CRISP/Cas-9. Error bars correspond to S.E.M., statistical analysis was performed using a Student's t-test (a-d) 1-way ANOVA with a Bonferroni post-hoc test (f and $\mathbf{h}$ ), or a 2-way ANOVA with a Bonferroni post-hoc test (g). n.s. means non-significant. ${ }^{* * *}$ and ${ }^{* * *}$ correspond to $P>0.05$, $P>0.01$ and $P>0.001$ respectively.

\section{Figure 2. GA but not DL rescues DJ-1 dependent sperm motility deficits and has an overall enhancing effect on sperm motility}

Box-and-Whisker plot graphics in $\mathbf{i}$ and $\mathbf{j}$ show the percentage of total motile (a) and progressive motile (b) sperm from WT and DJ-1 KO mice and the effect of GA and DL treatments on sperm motility. Box-and-Whisker plot graphic in (a) shows the percentage of progressively motile cryopreserved bull and human (fresh and cryopreserved) sperm 30 min after incubation in vehicle, 30 and 60 mM of GA. Graphics in ( $\mathbf{d}$ and $\mathbf{f}$ ) show the midterm effect of GA and DL on sperm motility in WT and DJ-1 mice. Graphics in (f , $\mathbf{g}$, and $\mathbf{h})$ show the midterm effect of GA on the motility of defrosted bull, fresh human and defrosted human sperm respectively. 2-way ANOVA: ctrl. vs. 30 or $60 \mathrm{mM} \mathrm{GA}$ in bull sperm ( $\mathrm{p}<0.001)$, ctrl. vs. 60 mM GA in human sperm (p<0.05), ctrl. vs. 30 mM GA (n.s.), ctrl. vs. 30 (n.s.) or $60 \mathrm{mM} \mathrm{GA}$ in cryopreserved human $(\mathrm{p}<0.05)$. . Error bars correspond to S.E.M., statistical analysis was performed using a 1-way ANOVA with a Bonferroni post-hoc test (a-c), or a 2-way ANOVA with a Bonferroni post-hoc 
test (d-h). n.s. means non-significant. ${ }^{*}, * *$ and ${ }^{* * *}$ correspond to $P>0.05, P>0.01$ and $P>0.001$ respectively.

Figure 3. GA and DL rescue DJ-1 and glod-4 dependent embryonic lethality in C. elegans and GA but not DL rescues DJ-1 dependent cell proliferation defects in HeLa cells Box-and-Whisker plot graphic in (a) display embryonic lethality in WT, $\Delta \Delta$ and glod-4 worms shown as the percentage of hatched eggs to the total of laid eggs and the effect of GA and DL treatment on embryonic lethality. (b) Effect of DJ-1 loss of function and the effect of GA and DL treatment on HeLa growth after 144 h. Error bars correspond to S.E.M., statistical analysis was performed using a 1-way ANOVA with a Bonferroni post-hoc test (a) or a Student's t-test (b).

Figure 4. GA slightly decreases mitochondrial membrane potential and calcium content in sperm cells but rescues the effect on motility of NNC550396 a CatSper inhibitor. Bar graphs in (a, b and $\mathbf{c})$ show the effect of GA treatment on $\Psi_{\mathrm{m}}$ on bull, fresh human and frozen human sperm respectively, measured as the percentage of sperm with JC-1 olig./JC-1 mon. values above a certain threshold (set as a gate in the FACS analysis).

Line graphic in (d) shows the effect of blocking CatSper with $1.5,3,5$, or $10 \mu \mathrm{M}$ NNC55-0396 and the co-treatment with GA on human progressive sperm motility. Statistical analysis was performed using 2-way ANOVA: Ctrl. vs. $3 \mu \mathrm{M}$ ( $\mathrm{p}<0.05)$, ctrl. vs. $5 \mu \mathrm{M}(\mathrm{p}<0.05)$, ctrl. vs. $3 \mu \mathrm{M}+\mathrm{GA}$ (n.s.), $3 \mu \mathrm{M}$ vs. $3 \mu \mathrm{M}$ GA (p<0.05), ctrl. vs. $5 \mu \mathrm{M}+\mathrm{GA}$ (n.s.), $5 \mu \mathrm{M}$ vs. $5 \mu \mathrm{M}+\mathrm{GA}$ (p<0.05), ctrl. vs. $10 \mu \mathrm{M}$ $(\mathrm{p}<0.05), 10 \mu \mathrm{M}$ vs. $10 \mu \mathrm{M}+\mathrm{GA}$ (n.s.). Box-and-Whisker plot graphic in (e) shows the effect of NNC55-0396 on bull sperm progressive motility and the enhancing effect of GA. Graphics in (f) to (h) show variations in the Cal-520-AM fluorescence signal upon treatment with $30 \mathrm{mM} \mathrm{GA}, 27 \mathrm{mM} \mathrm{NaCl}$ and $2 \mu \mathrm{M}$ progesterone in the absence of $\mathrm{Ca}^{2+}$ and after the addition of $\mathrm{Ca}^{2+}$ in human sperm (g) and upon treatment with $10 \mathrm{mM} \mathrm{GA}, 9 \mathrm{mM} \mathrm{NaCl}$ and $10 \mathrm{mM} 8$-Br-cAMP in the absence of $\mathrm{Ca}^{2+}$ and after the addition of $2 \mathrm{mM} \mathrm{Ca}^{2+}$ in sperm coming from WT (f) and CatSper KO (h) mice. Error bars correspond to S.E.M.. Statistical analysis was performed using a Student's t-test (a-c), 1-way ANOVA with a posthoc Bonferroni's multiple comparison test (e) or a 2-way ANOVA (d,f-h) were used. n.s. means non-significant. ${ }^{*}{ }^{* *}$ and ${ }^{* * *}$ correspond to $P>0.05, P>0.01$ and $P>0.001$ respectively.

Figure 5. GA but not DL increases NAD(P)H fluorescence in HeLa cells and both substances decrease the concentration of intracellular calcium.

Sequential microscope photos in (a) show the effect of GA and DL on NAD(P)H autofluorescence. Scale bar: $20 \mu \mathrm{M}$. As shown in graphic in (b), GA but not DL treatment increased NAD(P)H fluorescence when compared to control. 2-way ANOVA: Ctrl. vs. GA ( $p<0.001)$, ctrl. vs. DL (n.s.). Error bars correspond to S.E.M. 
Statistical analysis was performed using a 2-way ANOVA (b), a 1-way ANOVA with (c) or without (d) post-hoc Dunnett's test. n.s. means non-significant. *** and ${ }^{* * *}$ correspond to $P>0.05, P>0.01$ and $P>0.001$ respectively.

Figure 1

a

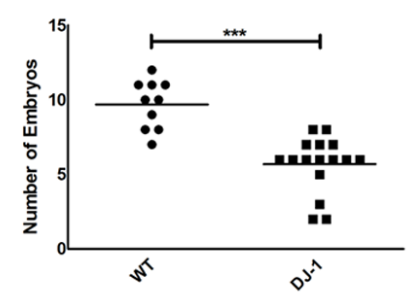

d

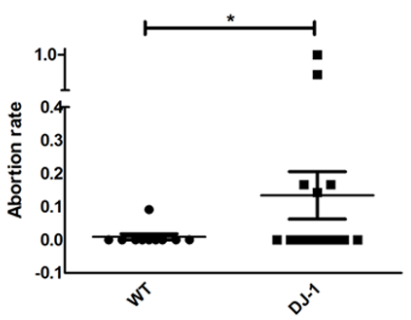

g

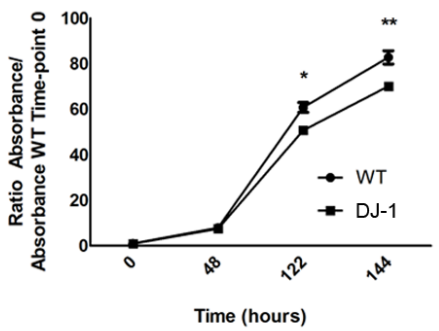

b

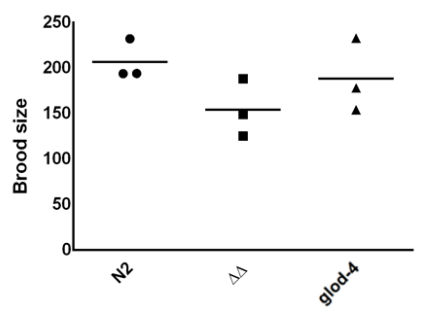

e

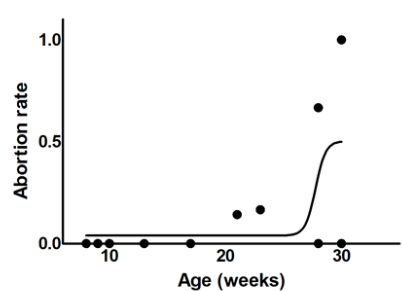

h

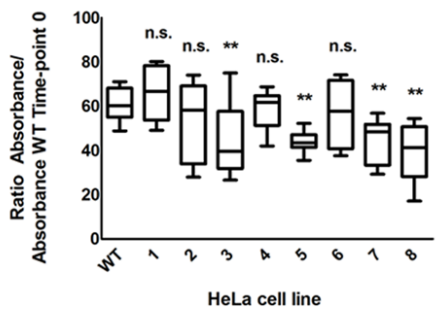

C

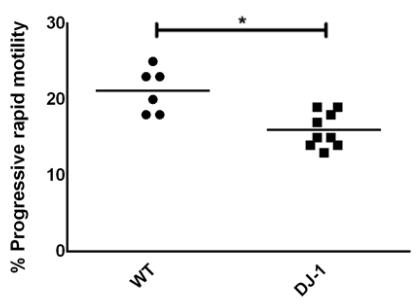

f

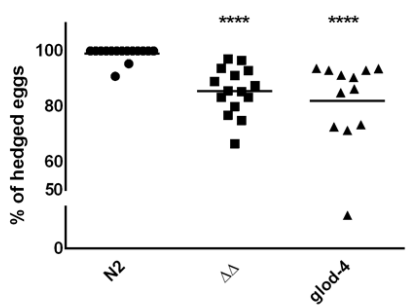


Figure 2
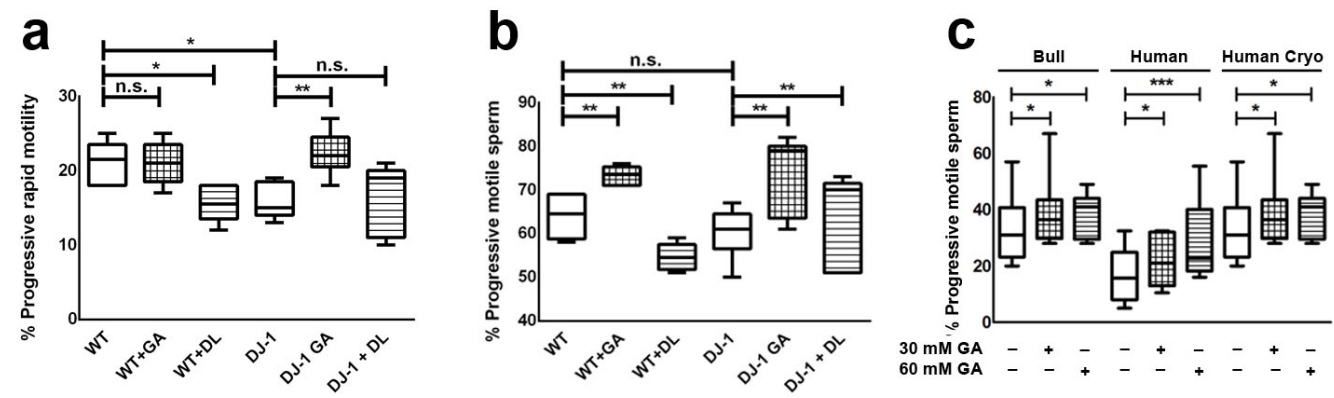

\section{d}

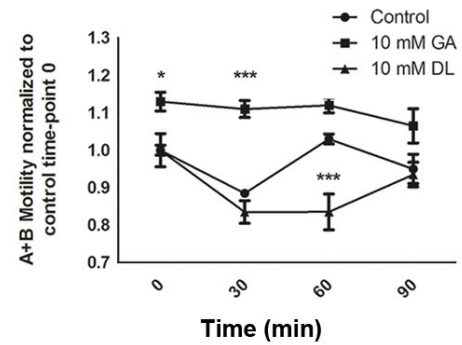

g

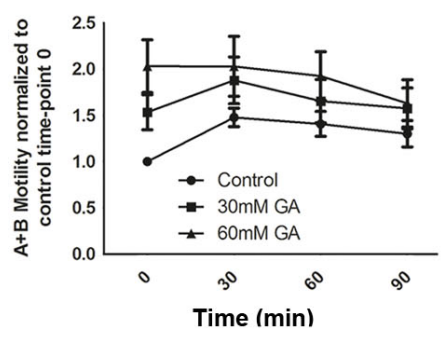

e

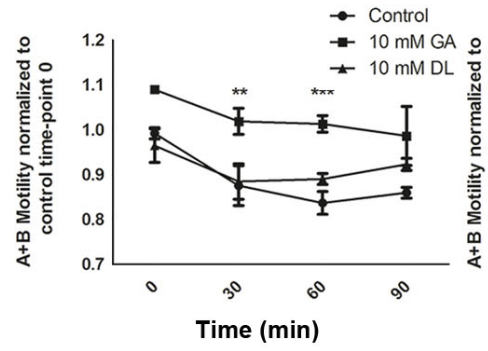

h

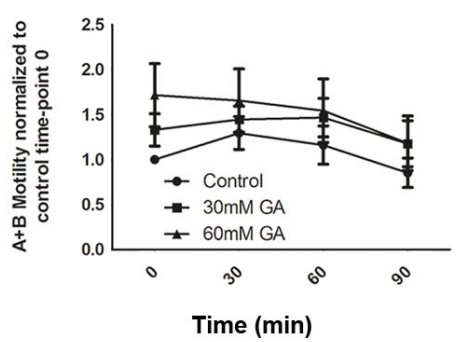

f

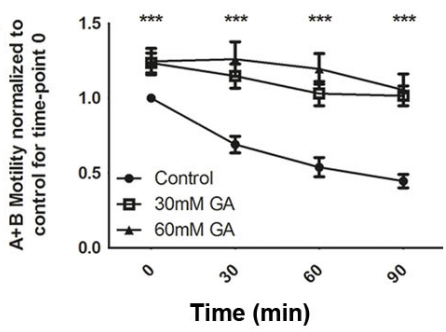

Time (min) 
Figure 3

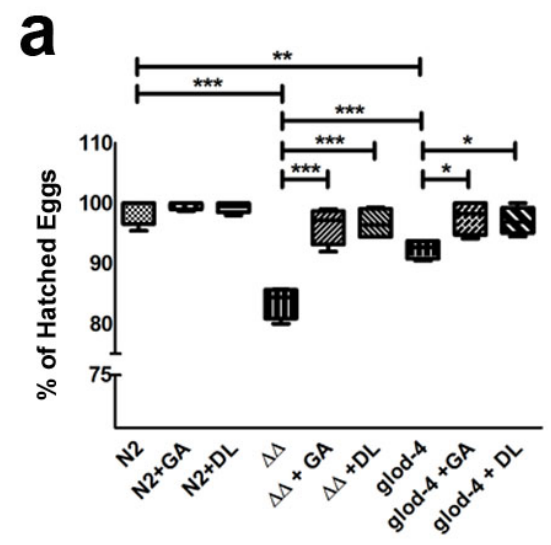

b

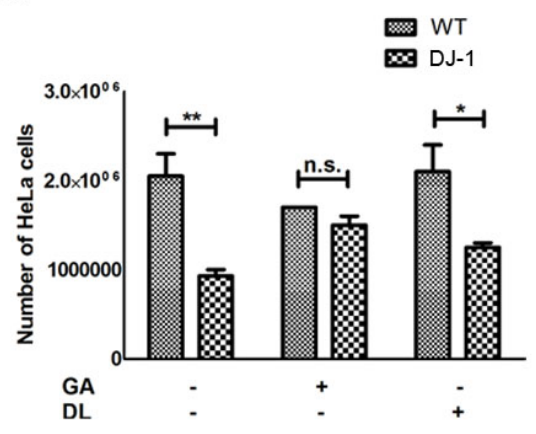


bioRxiv preprint doi: https://doi.org/10.1101/2021.01.16.426934; this version posted January 22, 2021. The copyright holder for this preprint (which was not certified by peer review) is the author/funder, who has granted bioRxiv a license to display the preprint in perpetuity. It is made available under aCC-BY-NC-ND 4.0 International license.

Figure 4

a

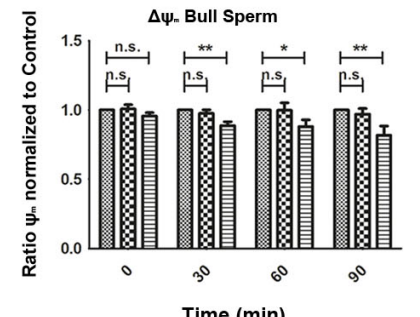

d

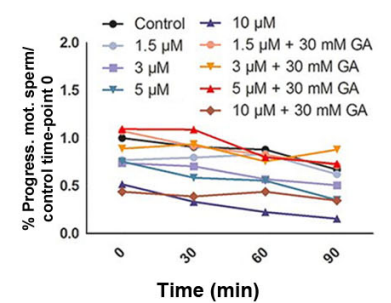

g

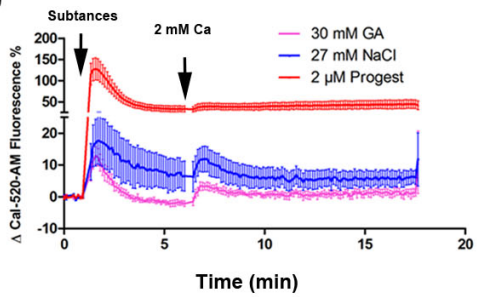

b

e
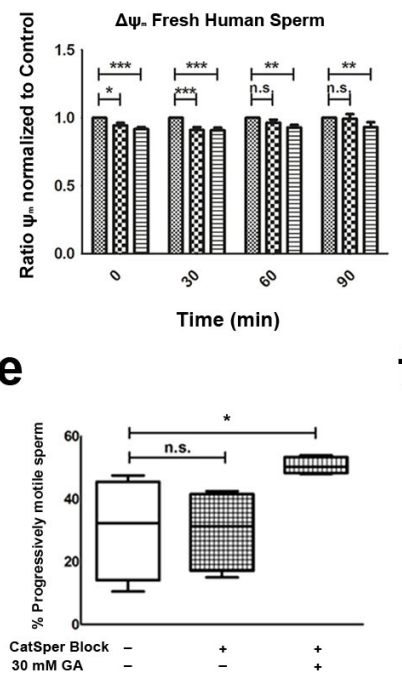

h CatSper KO Mice Sperm

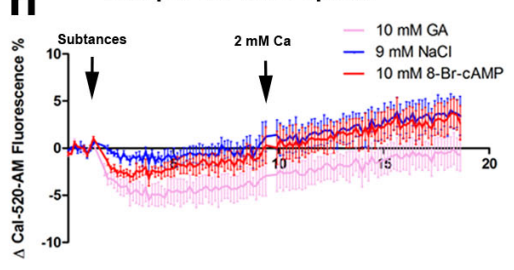

f

Time (min)
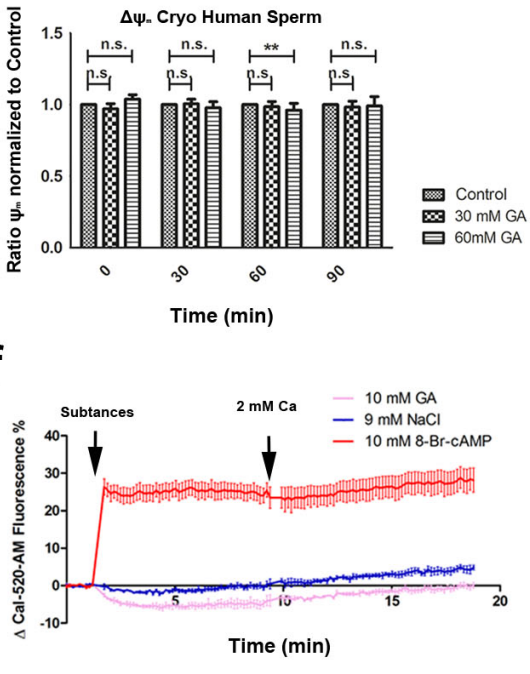
bioRxiv preprint doi: https://doi. org/10.1101/2021 01.16.426934 · this version posted January 22, 2021. The copyright holder for this

Figure 5

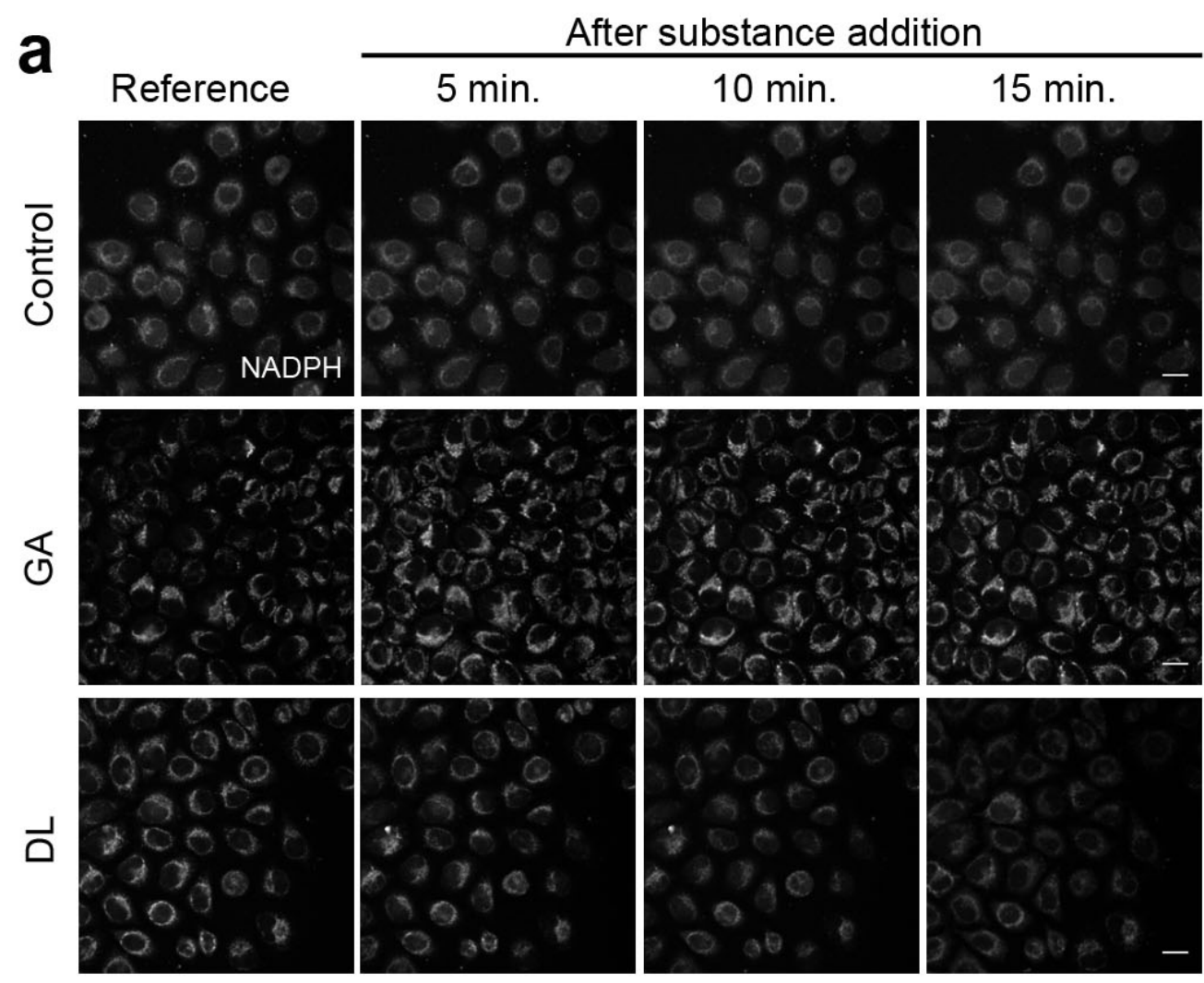

b

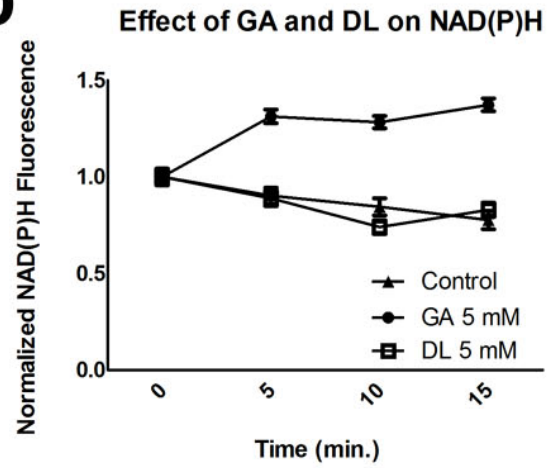

d

Fluo 4 DL
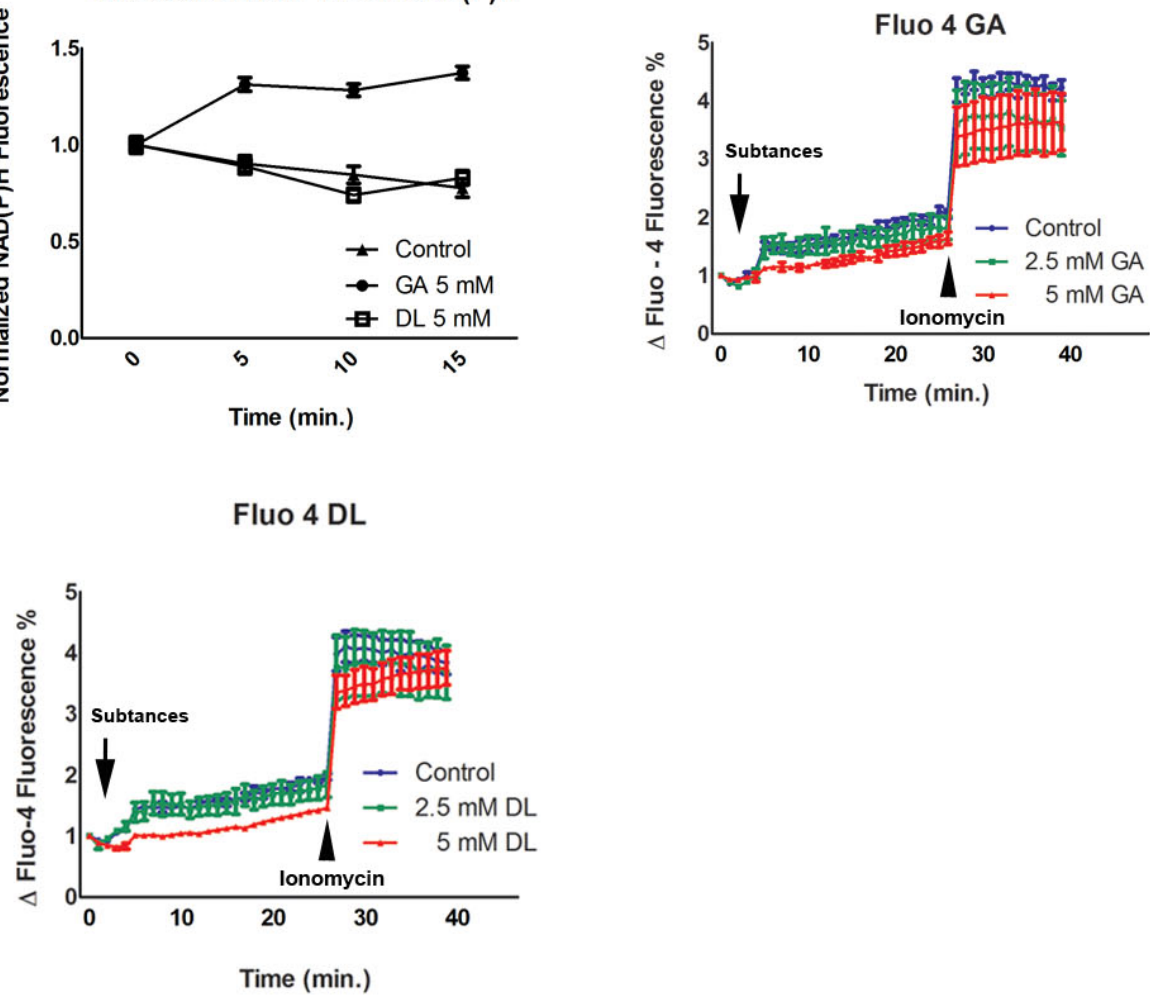